\title{
ANÁLISIS DEL COMPORTAMIENTO DEL SEGMENTO LUMBAR, NO ARTRODESADO, EN ESCOLIOSIS IDIOPÁTICA DEL ADOLESCENTE DE CURVA LENKE TIPO 1
}

\author{
ANÁLISE DO COMPORTAMENTO DO SEGMENTO LOMBAR SEM ARTRODESE EM \\ ESCOLIOSE IDIOPÁTICA DO ADOLESCENTE COM CURVATURA DE LENKE TIPO 1
}

\begin{abstract}
ANALYSIS OF THE BEHAVIOR OF THE LUMBAR SEGMENT WITHOUT SPINAL FUSION IN ADOLESCENT IDIOPATHIC SCOLIOSIS WITH CURVATURE OF LENKE TYPE 1
\end{abstract}

Nicolás Siderakis ${ }^{1}$, Ariel Aguilar² ${ }^{2}$ Dino Dinelli ${ }^{2}$, Lázaro Lutzky ${ }^{3}$, Juan Reviriego ${ }^{4}$, María Escalada ${ }^{4}$, Gabriel Rositto ${ }^{5}$, Carlos Legarreta ${ }^{6}$

\section{RESUMEN}

Objetivo: Determinar el comportamiento del segmento lumbar, no artrodesado, en el plano coronal luego de fusiones torácicas selectivas en pacientes con escoliosis idiopática del adolescente con curvas Lenke 1. Métodos: Se evaluaron 21 pacientes con un seguimiento promedio de 29,71 meses (rango: 24-60 meses) mediante análisis clínico y radiográfico retrospectivos de pacientes que presentaron escoliosis idiopáticas del adolescente, con curvas Lenke tipo 1 (ABC). Resultados: En todos los pacientes, en los que se produjo un desequilibrio en el plano coronal, se constató la progresión del valor angular de la curva lumbar no artrodesada al final del seguimiento. Conclusión: La curva lumbar no artrodesada progresó al final del seguimiento, en todos los pacientes con desequilibrio confirmado en el plano coronal (3/21).

Descriptores: Región lumbosacra; Escoliosis; Fusión vertebral; Adolescente.

\section{RESUMO}

Objetivo: Determinar o comportamento do segmento lombar sem artrodese no plano coronal, após fusões torácicas seletivas na escoliose idiopática do adolescente com curvaturas de Lenke 1. Métodos: Foram avaliados 21 pacientes com acompanhamento médio de 29,71 meses (faixa: 24 a 60 meses), por meio de análise clínica e radiográfica retrospectiva de pacientes que apresentaram escoliose idiopática do adolescente com curvaturas de Lenke tipo 1 (ABC). Resultados: Constatou-se aumento do ângulo da curva lombar sem artrodese no final do acompanhamento em todos os pacientes que apresentaram desequilíbrio no plano coronal. Conclusão: O total dos pacientes nos quais se confirmou desequilíbrio no plano coronal (3/21) apresentaram progressão da curva lombar sem artrodese no final do acompanhamento.

Descritores: Região lombossacral; Escoliose; Fusão vertebral; Adolescente.

\begin{abstract}
Objective: To determine the outcome of lumbar segment without arthrodesis in the coronal plane, after selective thoracic fusions for with curvature of Lenke type 1 adolescent idiopathic scoliosis with curvature of Lenke type 1. Methods: Twenty one patients were evaluated, with a mean follow-up of 29.71 months (range: 24-60 months) with retrospective clinical and radiographic analysis of patients with adolescent idiopathic scoliosis with curvature of Lenke type 1 $(A B C)$. Results: The increase of the angle of the lumbar curve without fusion was documented at the end of the follow-up in patients with coronal plane imbalance. Conclusion: Lumbar curve without fusion progressed at the end of the follow-up in patients with documented coronal plane imbalance (3/21).
\end{abstract}

Keywords: Lumbosacral region; Scoliosis; Spinal fusion; Adolescent.

\section{INTRODUCCIÓN}

En la actualidad, el manejo de las escoliosis idiopáticas torácicas asociadas a una curva lumbar no estructurada genera controversias para determinar el nivel distal de la fusión ${ }^{1}$. El objetivo del cirujano espinal es ahorrar niveles de artrodesis, mantener el balance de la columna con movilidad y disminuir la incidencia de la degeneración discal en la vida adulta ${ }^{2,3}$. El consenso general indica que se acepta la fusión selectiva torácica en deformidades con curvas lumbares flexibles (tipo Lenke 1 con modificadores A y B), pero aún hay controversias en las de tipo $C^{4}$. El número de estudios con un seguimiento prolongado es limitado. Aunque la fusión selectiva tiene la ventaja de preservar la movilidad de los segmentos lumbares, en algunos casos, podría producirse la descompensación posoperatoria en el plano coronal $1^{1,2,5}$. Teniendo en cuenta esto, el desafío es pronosticar si el comportamiento del segmento lumbar no fusionado será agresivo o no evolutivo. Para tal fin, se determinarán una serie de elementos que ayudarán a descifrar dicho comportamiento.

\section{MATERIAL Y MÉTODOS}

Estudio retrospectivo clínico y radiográfico de tipo serie de casos. Se evaluaron 21 pacientes, con un seguimiento promedio de 29.71 meses (rango: de 24 a 60 meses) mediante el análisis clínico y radiográfico retrospectivo de pacientes con escoliosis idiopática

\footnotetext{
1. Médico concurrente.

2. Fellowship, Servicio de Patologia Espinal del Hospital Pediatrico Dr. R. Gutierrez.

3. Médico concurrente, Servicio de Patologia Espinal del Hospital Pediatrico Dr. R. Gutierrez.

4. Médico(a) de planta, Servicio de Patologia Espinal del Hospital Pediatrico Dr. R. Gutierrez.

5. Sub-Jefe de Servicio de Patologia Espinal del Hospital Pediatrico Dr. R. Gutierrez.

6. Jefe de Servicio de Patologia Espinal del Hospital Pediatrico Dr. R. Gutierrez.
}

Trabajo realizado: Sección Patología de la Columna Vertebral - Hospital de Niños “Ricardo Gutiérrez Gallo 1330 (1425) Ciudad Autónoma de Buenos Aires, República Argentina. Correspondencia: Av. Diaz Vélez 4760 Piso 8 “D”. nicolassiderakis@yahoo.com.ar 
del adolescente, con curvas Lenke tipo 1 (ABC) (Tabla 1). La edad promedio al momento de cirugía fue 17.05 años (rango: de 13 a 23 años); la distribución por sexo fue 18 mujeres y 3 hombres.

\section{Criterios de inclusión}

Pacientes con diagnóstico de escoliosis idiopática del adolescente Lenke tipo 1, en los cuales se evaluó el comportamiento de la curva lumbar no fusionada, con un seguimiento mínimo de dos años.

\section{Parámetros clínicos analizados}

- Menarca al momento de la cirugía;

- Signo de Adams;

- Equilibrio del tronco.

\section{Parámetros radiológicos}

- Espinograma de frente y perfil, de pie; preoperatorio y a los 30 días, 3 meses, 6 meses de la cirugía, y en el último control (más de dos años);

- Medición del valor angular (Cobb) (Tabla 2);

- Rotación vertebral (Nash-Moe);

- Modificador lumbar (Lenke);

- Radiografías en flexión lateral y tracción, para evaluar el porcentaje de flexibilidad de las curvas.

\section{Análisis de las técnicas de tratamiento empleadas:}

- Vías de abordaje;

- Sistemas híbridos (ganchos/tornillos);

- Sistema de fijación transpedicular;

- Métodos de corrección (desrotación global de la curva/cantilever); - Toracoplastia.

Tabla 1. Resultados del análisis del modificador lumbar.

\begin{tabular}{c|c}
\hline Modificador lumbar A & 6 pacientes \\
\hline Modificador lumbar B & 7 pacientes \\
\hline Modificador lumbar C & 8 pacientes \\
\hline
\end{tabular}

Tabla 2. Valor angular preoperatorio, plano coronal.

\begin{tabular}{c|c}
\hline Curva torácica & Resultados \\
\hline Valor angular global & $57,35^{\circ}\left(\mathrm{R}:\right.$ de $40^{\circ}$ a $\left.84^{\circ}\right)$ \\
\hline Porcentaje de corrección (bending) & $40,9 \%(\mathrm{R}:$ del $4 \%$ al $59,9 \%)$ \\
\hline Curva lumbar & Resultados \\
\hline Valor angular global & $37,95^{\circ}\left(\mathrm{R}:\right.$ de $27^{\circ}$ a $\left.50^{\circ}\right)$ \\
\hline Porcentaje de corrección (bending) & $76 \%(\mathrm{R}$ : del $56,5 \%$ al $100 \%)$ \\
\hline
\end{tabular}

\section{RESULTADOS}

Se evaluó a 21 pacientes (18 mujeres y 3 varones), el promedio de edad al momento de la cirugía era de 17.5 años.

En la Tabla 3, se detallan los resultados del análisis inicial de la evolución de los segmentos artrodesados y no artrodesados.

El promedio global del valor angular de las curvas torácicas artrodesadas a los 6 meses de seguimiento posoperatorio fue de $25^{\circ}$, que corresponde a un porcentaje global promedio de corrección posquirúrgica del 43,59\%.

Se realizó una evaluación prequirúrgica clínica y radiográfica del balance coronal general de los pacientes, y se constató que cuatro tenían un balance coronal neutro (19\%); 8 , un desequilibrio coronal hacia la derecha (38\%); y 9, un desequilibrio hacia la izquierda (43\%) (Tabla 4).

De los 9 pacientes con un desequilibrio lateral del tronco prequirúrgico hacia la izquierda, en 6 de ellos, se documentó la progresión del valor angular de la curva lumbar no artrodesada, al final del seguimiento. Por el contrario, de los 12 pacientes con balances coronales prequirúrgicos neutros o desequilibrio hacia la derecha, solo 4 tenían progresión de la curva lumbar no artrodesada.

Entre los pacientes en quienes se constató desequilibrio del tronco en el plano coronal, dos tenían desequilibrio hacia la izquierda y uno neutro. Teniendo en cuenta esto, se realizó el análisis de la corrección posquirúrgica de la curva torácica alcanzada en estos pacientes. El promedio de corrección posquirúrgica de las curvas torácicas en los pacientes con un desequilibrio coronal fue del $55,46 \%$.

En los pacientes que tuvieron un desequilibrio en el plano coronal, se comprobó la progresión del valor angular de la curva lumbar no artrodesada al final del seguimiento.
Tabla 3. Evolución posoperatoria, plano coronal - Curva torácica.

\begin{tabular}{|c|c|c|c|c|}
\hline & Modificador A & Modificador B & Modificador C & \\
\hline A los 30 días & & & & 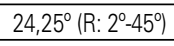 \\
\hline A los 3 meses & & & & \\
\hline A los $6 \mathrm{~m}$ & & & & \\
\hline & & & & \\
\hline & $20,5^{\circ}\left(\mathrm{R}: 10^{\circ}-45^{\circ}\right)$ & & & \\
\hline \multicolumn{5}{|c|}{ Evolucion posoperatoria, plano coronal - Curva lumbar } \\
\hline & & & & \\
\hline Alos & & & 24,30 & $16,5^{\circ}$ \\
\hline$A \operatorname{los} 3$ & & $\left.-28^{\circ}\right)$ & $\left.-35^{\circ}\right)$ & $16,9^{\circ}($ \\
\hline$\overline{A \operatorname{los} 6}$ & $\left.-30^{\circ}\right)$ & $\left.-29^{\circ}\right)$ & 22,7 & $17,57^{\circ}$ \\
\hline & & & $28,25^{\circ}$ & $22^{\circ}(\mathrm{R}:$ \\
\hline A los 2 años & $14,66^{\circ}\left(\mathrm{R}: 5^{\circ}-35^{\circ}\right)$ & $25,66^{\circ}\left(\mathrm{R}: 14^{\circ}-35^{\circ}\right)$ & $33,8^{\circ}\left(R: 8^{\circ}-40^{\circ}\right)$ & $24,17^{\circ}\left(\mathrm{R}: 8^{\circ}-40^{\circ}\right.$ \\
\hline
\end{tabular}

Tabla 4. Analisis evolutivo del balance coronal general.

\begin{tabular}{|c|c|c|c|}
\hline & Prequirúrgico & 6 meses & 2 años \\
\hline 1 & $1 \mathrm{~cm} \mathrm{IZO}$ & Neutro & $0,5 \mathrm{~cm}$ IZO \\
\hline 2 & Neutro & 2,3 cm DER & $0,5 \mathrm{~cm}$ DER \\
\hline 3 & $1,3 \mathrm{~cm}$ IZO & $1,5 \mathrm{~cm} \mathrm{IZO}$ & $1,7 \mathrm{~cm} \mathrm{IZO}$ \\
\hline 4 & $1,5 \mathrm{~cm}$ DER & Neutro & $0,7 \mathrm{~cm}$ DER \\
\hline 5 & $1 \mathrm{~cm} \mathrm{DER}$ & Neutro & Neutro \\
\hline 6 & $1,4 \mathrm{~cm}$ DER & $0,5 \mathrm{~cm}$ DER & Neutro \\
\hline 7 & $1 \mathrm{~cm} \mathrm{IZO}$ & $1,7 \mathrm{~cm} \mathrm{IZO}$ & $2,7 \mathrm{~cm} \mathrm{IZO}$ \\
\hline 8 & $1,7 \mathrm{~cm} \mathrm{IZO}$ & $2 \mathrm{~cm} \mathrm{IZO}$ & $2,5 \mathrm{~cm}$ IZO \\
\hline 9 & Neutro & $1,6 \mathrm{~cm} \mathrm{IZO}$ & $1,5 \mathrm{~cm} \mathrm{IZO}$ \\
\hline 10 & $1,5 \mathrm{~cm} \mathrm{IZO}$ & $0.5 \mathrm{~cm} \mathrm{IZO}$ & Neutro \\
\hline 11 & $1.3 \mathrm{~cm}$ DER & Neutro & $0,6 \mathrm{~cm}$ DER \\
\hline 12 & Neutro & $1 \mathrm{~cm} \mathrm{DER}$ & $2 \mathrm{~cm} \mathrm{DER}$ \\
\hline 13 & $1 \mathrm{~cm} \mathrm{IZQ}$ & Neutro & Neutro \\
\hline 14 & Neutro & Neutro & Neutro \\
\hline 15 & $1,1 \mathrm{~cm} \mathrm{IZO}$ & Neutro & Neutro \\
\hline 16 & $1,5 \mathrm{~cm} \mathrm{IZO}$ & $1,5 \mathrm{~cm} \mathrm{IZO}$ & Neutro \\
\hline 17 & $2,5 \mathrm{~cm} \mathrm{DER}$ & $1,5 \mathrm{~cm} \mathrm{DER}$ & $1 \mathrm{~cm} \mathrm{DER}$ \\
\hline 18 & $2 \mathrm{~cm} \mathrm{DER}$ & $1 \mathrm{~cm} \mathrm{DER}$ & $1,5 \mathrm{~cm}$ DER \\
\hline 19 & $1,2 \mathrm{~cm}$ DER & $0,6 \mathrm{~cm}$ DER & Neutro \\
\hline 20 & $1,5 \mathrm{~cm} \mathrm{DER}$ & Neutro & Neutro \\
\hline 21 & $1 \mathrm{~cm} \mathrm{IZQ}$ & $0,5 \mathrm{~cm}$ IZO & $1,4 \mathrm{~cm}$ IZO \\
\hline
\end{tabular}

Al efectuar un análisis de los niveles de artrodesis en nuestra serie, el promedio fue de 8,5 niveles, con un rango de 8 a 12 niveles; el segmento anatómico T5-L1 fue el sitio más frecuente. Tras el análisis discriminado, se halló que se realizó una instrumentación Cobb +1 en 5 pacientes, Cobb +2 en 8 pacientes y Cobb a Cobb en 8 pacientes.

Se analizó la evolución de la rotación vertebral (Tabla 5) de la curva lumbar no artrodesada realizando un promedio, discriminando aquellas en las que progresó el valor angular y las curvas en las que se constató la corrección espontánea.

Se calculó el ratio entre Cobb torácico/Cobb lumbar de todos los pacientes. El ratio global fue de 1,5. Se considera que, para una fusión selectiva, debe ser $>1,2$. Solo un paciente tuvo un ratio $<1,2(1,17)$; en ese caso, se verificó la progresión de la curva lumbar no artrodesada.

En 10 pacientes, se detectó la progresión del valor angular de la curva lumbar no fusionada, el promedio en grados fue de $9,1^{\circ}$ (rango: de $6^{\circ}$ a $1^{\circ}$ ) en dos años. En 8 de ellos, se observó la progresión en el primer año de seguimiento posoperatorio (Tabla 6 y Figura $1 \mathrm{~A}, \mathrm{~B}$ e $\mathrm{C}$ )

Se documentó la corrección espontánea de la curva lumbar no fusionada, con un promedio de 18,55 (Tabla 7), en 11 pacientes.

En la Tabla 8, se detallan los resultados de las curvas con corrección espontánea, teniendo en cuenta la discriminación por modificador lumbar.

Tres pacientes sufrieron complicaciones: uno presentó pull-out de tornillos con pérdida de carga distal; otro, pull-out de tornillos distales derechos con pérdida de corrección de ambas curvas, sin requerir extensión de artrodesis; y otro necesitó una extensión de la artrodesis a nivel lumbar por descompensación grave del tronco.

En todos los pacientes con complicaciones, se constató la progresión de la curva lumbar o torácica en el primer año de seguimiento. 
Tabla 5. Analisis de la rotación vertebral del segmento no artrodesado.

\begin{tabular}{l|c|c}
\hline & $\begin{array}{c}\text { Rotación } \\
\text { prequirúrgica }\end{array}$ & $\begin{array}{c}\text { Rotación al final del } \\
\text { seguimiento }\end{array}$ \\
\hline Curvas lumbares que progresaron & 2,7 & 2,22 \\
\hline Curvas lumbares que se corrigieron & 1,9 & 1,18 \\
\hline
\end{tabular}

Tabla 6. Progresión valor angular del segmento lumbar no artrodesado.

\begin{tabular}{c|c|c|c|c|c}
\hline & Paciente & 30 días & 6 meses & 1 año & 2 años \\
\hline 1 & $\mathrm{~L} 1 \mathrm{CN}$ & $20^{\circ}$ & $23^{\circ}$ & $30^{\circ}$ & $30^{\circ}$ \\
\hline 2 & $\mathrm{~L} 1 \mathrm{C}-$ & $25^{\circ}$ & $35^{\circ}$ & $39^{\circ}$ & $39^{\circ}$ \\
\hline 3 & $\mathrm{~L} 1 \mathrm{~B}-$ & $22^{\circ}$ & $25^{\circ}$ & $31^{\circ}$ & $30^{\circ}$ \\
\hline 4 & $\mathrm{~L} 1 \mathrm{CN}$ & $21^{\circ}$ & $23^{\circ}$ & $30^{\circ}$ & $30^{\circ}$ \\
\hline 5 & $\mathrm{~L} 1 \mathrm{~B}-$ & $28^{\circ}$ & $29^{\circ}$ & $31^{\circ}$ & $35^{\circ}$ \\
\hline 6 & $\mathrm{~L} 1 \mathrm{~A}+$ & $27^{\circ}$ & $30^{\circ}$ & $35^{\circ}$ & $35^{\circ}$ \\
\hline 7 & $\mathrm{~L} 1 \mathrm{BN}$ & $13^{\circ}$ & $13^{\circ}$ & $25^{\circ}$ & $28^{\circ}$ \\
\hline 8 & $\mathrm{~L} 1 \mathrm{~B}+$ & $5^{\circ}$ & $18^{\circ}$ & $18^{\circ}$ & $20^{\circ}$ \\
\hline 9 & $\mathrm{~L} 1 \mathrm{~A}+$ & $2^{\circ}$ & $10^{\circ}$ & $10^{\circ}$ & $10^{\circ}$ \\
\hline 10 & $\mathrm{~L} 1 \mathrm{C}+$ & $35^{\circ}$ & $34^{\circ}$ & $35^{\circ}$ & $40^{\circ}$ \\
\hline
\end{tabular}
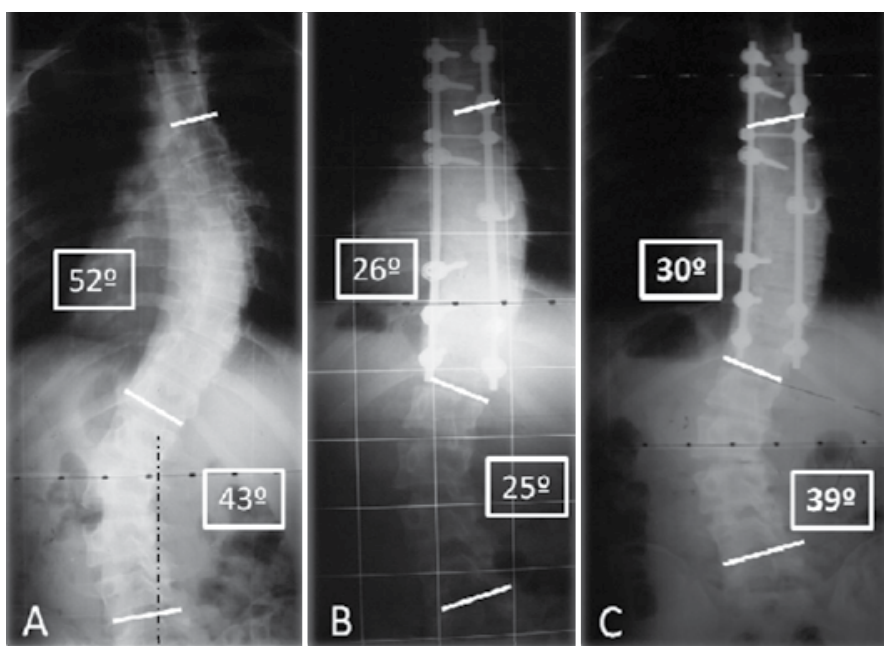

Figura 1. (A) Paciente ZN de sexo masculino, 15 años de edad, con escoliosis idiopática del adolescente con una curva torácica derecha de $52^{\circ}$ estructurada y una curva lumbar izquierda de $43^{\circ}$ no estructurada. Modificador lumbar C. Se practicó instrumentación T4-T12 por vía posterior. (B) A los seis meses de la cirugía, se constata la disminución del valor angular de ambas curvas. (C) A año, se documenta la progresión de $14^{\circ}$ del segmento lumbar no artrodesado acompañada de una progresión del valor angular del segmento artrodesado.

\section{DISCUSIÓN}

El objetivo principal de la fusión torácica selectiva es tratar la deformidad torácica sin comprometer la movilidad lumbar; en muchos casos, se observa la corrección espontánea de la deformidad de la curva lumbar ${ }^{5}$. La controversia surge, porque no es posible la fusión torácica selectiva en todos los casos, con el potencial riesgo de la descompensación de la curva y el desequilibrio del tronco. Las recomendaciones iniciales de King eran que la fusión caudal se extendiera hasta la primera vértebra estable, tomando los modifica-
Tabla 7. Corrección espontanea del segmento lumbar no artrodesado.

\begin{tabular}{c|c|c|c|c}
\hline & Paciente & Prequirúrgico & 6 meses & 2 años \\
\hline 1 & L1AN & $27^{\circ}$ & $10^{\circ}$ & $15^{\circ}$ \\
\hline 2 & L1A- & $45^{\circ}$ & $10^{\circ}$ & $5^{\circ}$ \\
\hline 3 & L1AN & $34^{\circ}$ & $9^{\circ}$ & $8^{\circ}$ \\
\hline 4 & L1C- & $35^{\circ}$ & $33^{\circ}$ & $27^{\circ}$ \\
\hline 5 & L1BN & $50^{\circ}$ & $24^{\circ}$ & $27^{\circ}$ \\
\hline 6 & L1BN & $28^{\circ}$ & $12^{\circ}$ & $11^{\circ}$ \\
\hline 7 & L1CN & $38^{\circ}$ & $25^{\circ}$ & $28^{\circ}$ \\
\hline 8 & L1C- & $30^{\circ}$ & $10^{\circ}$ & $14^{\circ}$ \\
\hline 9 & L1C- & $33^{\circ}$ & $17^{\circ}$ & $18^{\circ}$ \\
\hline 10 & L1B- & $44^{\circ}$ & $22^{\circ}$ & $20^{\circ}$ \\
\hline 11 & L1A+ & $32^{\circ}$ & $14^{\circ}$ & $17^{\circ}$ \\
\hline & & & &
\end{tabular}

Tabla 8. Grado de corrección angular lumbar según modificador.

\begin{tabular}{c|c|c|c}
\hline & Modificador A & Modificador B & Modificador C \\
\hline Promedio porcentaje de corrección & $64,21 \%$ & $56,41 \%$ & $34,94 \%$ \\
\hline Promedio en grados de corrección & $23,25^{\circ}$ & $21^{\circ}$ & $13,25^{\circ}$ \\
\hline
\end{tabular}

dores $\mathrm{B}$ y $\mathrm{C}$ como variantes graves ${ }^{1}$. Las recomendaciones actuales tienden a otorgarle al cirujano las herramientas necesarias para predecir la evolución de la curva lumbar no fusionada. Algunos de los factores citados como causas de la descompensación lumbar son hipercorrección de la curva torácica ${ }^{6}$, elección de los niveles de fusión, analizada, entre otros, por Suk et al. ${ }^{7}$, incorrecta interpretación de los patrones de curva, análisis incorrecto de la curva lumbar y rotación de vértebras apicales ${ }^{5}$. Sucato comunica que uno de los parámetros radiológicos que considera para determinar la fusión selectiva es el ratio de la magnitud de curva torácica sobre el de la curva lumbar, que tiene que ser $>1,2^{4}$. A su vez, analiza dos parámetros radiológicos de importancia para definir una fusión selectiva: el balance coronal, si es neutro o hacia la derecha, curva lumbar no estructurada, indica fusión selectiva; en cambio, si el shift es hacia la izquierda la contraindica. Otro parámetro es el la inclinación del platillo superior de L4 que, si es hacia la izquierda, indica fusión selectiva, si es neutro o hacia la derecha contraindica la fusión selectiva ${ }^{8}$.

\section{CONCLUSIÓN}

En la actualidad, uno de los puntos de controversia es saber cuál es el comportamiento de la curva lumbar no artrodesada en pacientes con curvas de tipo Lenke 1. De acuerdo con el estudio realizado en nuestra serie, es posible concluir que:

- Todos los pacientes en los que se constató desequilibrio en el plano coronal (3/21) tuvieron progresión de la curva lumbar no artrodesada al final del seguimiento.

- Los pacientes con un balance coronal preoperatorio del tronco hacia la izquierda (66\%) sufrieron progresión de la curva lumbar no artrodesada. - La capacidad de la curva lumbar no artrodesada de realizar la corrección espontánea de la deformidad es menor, a medida que se progresa en el modificador lumbar.

- Al analizar la rotación vertebral del segmento no artrodesado, esta disminuyó globalmente al final del seguimiento.

- La hipolordosis posoperatoria fue un factor predisponente en las curvas lumbares que progresaron.

\section{REFERENCIAS}

1. Chang MS, Bridwell KH, Lenke LG, Cho W, Baldus C, Auerbach JD, et al. Predicting the outcome of selective thoracic fusion in false double major lumbar " $C$ " cases with five- to twenty-four-year follow-up. Spine (Phila Pa 1976). 2010;35(24):2128-33.

2. Sucato DJ. Selective versus nonselective surgery for adolescent idiopathic scoliosis. In: Newton PO, O'Brien MF, Shufflebarger HL, Betz RR, Dickson RA, Harms J, editors. Idiopathic scoliosis. The harms study group treatment guide. New York: Thieme Medical Publishers; 2010. p. 150

3. Edwards CC 2nd, Lenke LG, Peelle M, Sides B, Rinella A, Bridwell KH. Selective thoracic fusion for adolescent idiopathic scoliosis with $\mathrm{C}$ modifier lumbar curves: 2- to 16-year radiographic and clinical results. Spine (Phila Pa 1976). 2004:29(5):536-46.

4. Winter RB, Lonstein JE. A meta-analysis of the literature on the issue of selective thoracic fusion for the King-Moe type II curve pattern in adolescent idiopathic scoliosis. Spine (Phila Pa 1976). 2003;28(9):948-52

5. Dobbs MB, Lenke LG, Walton T, Peelle M, Della Rocca G, Steger-May K, et al. Can we predict the ultimate lumbar curve in adolescent idiopathic scoliosis patients undergoing a selective fusion with undercorrection of the thoracic curve? Spine (Phila Pa 1976) 2004:29(3):277-85.

6. Imrie M, Yaszay B, Bastrom TP, Wenger DR, Newton PO. Adolescent idiopathic scoliosis: should 100\% correction be the goal? J Pediatr Orthop. 2011;31(Suppl1):S9-13.

7. Suk SI, Lee SM, Chung ER, Kim JH, Kim WJ, Sohn HM. Determination of distal fusion level with segmental pedicle screw fixation in single thoracic idiopathic scoliosis. Spine (Phila Pa 1976). 2003:28(5):484-91.

8. Miyanji F, Pawelek JB, Van Valin SE, Upasani V, Newton PO. Is the lumbar modifier useful in surgical decision making?: defining two distinct Lenke 1 A curve patterns. Spine (Phila Pa 1976). 2008;33(23):2545-51. patterns. 